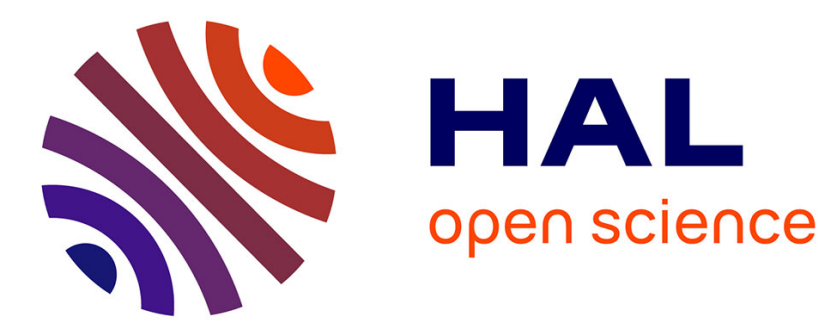

\title{
Ultrasonic Computed Tomography
}

Philippe Lasaygues, Régine Guillermin, Jean-Pierre Lefebvre

\section{To cite this version:}

Philippe Lasaygues, Régine Guillermin, Jean-Pierre Lefebvre. Ultrasonic Computed Tomography. P. Laugier, G. Haiat. Bone Quantitative Ultrasound, Springer, pp.441-459, 2010. hal-00492836

\section{HAL Id: hal-00492836 https://hal.science/hal-00492836}

Submitted on 17 Jun 2010

HAL is a multi-disciplinary open access archive for the deposit and dissemination of scientific research documents, whether they are published or not. The documents may come from teaching and research institutions in France or abroad, or from public or private research centers.
L'archive ouverte pluridisciplinaire HAL, est destinée au dépôt et à la diffusion de documents scientifiques de niveau recherche, publiés ou non, émanant des établissements d'enseignement et de recherche français ou étrangers, des laboratoires publics ou privés. 


\title{
18. Ultrasonic Computed Tomography
}

\author{
Philippe Lasaygues, Régine Guillermin, Jean-Pierre Lefebvre
}

Laboratoire de Mécanique et d'Acoustique, UPR CNRS 7051 31, chemin Joseph Aiguier, 13402 Marseille cedex 20 - France

\begin{abstract}
Ultrasonic Computed Tomography (UCT) is a full digital imaging technique, which consists in numerically solving the inverse scattering problem associated to the forward scattering problem describing the interaction of ultrasonic waves with inhomogeneous media. For weakly inhomogeneous media such as soft tissues, various approximations of the solution of the forward problem (straight ray approximation, Born approximation...), leading to easy-to-implement approximations of the inverse scattering problem (back-projection or backpropagation algorithms) can be used. In the case of highly heterogeneous media such as bone surrounded by soft tissues, such approximations are no more valid. We present here two non-linear inversion schemes based on high-order approximations. These methods are conceived like the prolongation of the methods implemented in the weakly inhomogeneous case for soft tissues. The results show the feasibility of this UCT approach to bones and its potential to perform measurements in vivo.
\end{abstract}

\section{Introduction}

Clinical ultrasound, which was developed for soft biological tissues, i.e., weakly inhomogeneous media, is not suitable for studying hard tissues such as bone because of the high acoustical properties contrast existing between the bone and the surrounding soft tissues. Some promising attempts were achieved by combining the mechanical displacement of mono-element ultrasonic probes with numerical processing of B-mode images, known as ultrasonic echo-tomography, for example on the brain [1] and long bones [2]. All these methods result in qualitative images of the internal structure of the imaged media, but they fail to provide quantitative estimates of relevant physical parameters, such as the velocity or the attenuation of the ultrasonic wave, or the acoustical impedance of the medium. Ultrasonic Computed Tomography (UCT), which combines $X$-ray Computed Tomography $(C T)$ reconstruction procedures (back-projection or backpropagation algorithms) and ultrasonic waves, is expected to yield parametric cross-sectional images, i.e., images of wave velocities, wave attenuations or acoustical impedances. $U C T$ has been developed by several authors to study to 
soft tissues $[3,4,5,6]$, using approaches such as the straight rays approximation [7] or the Born approximation [8], the latter consisting in assuming that the total field is equal to the incident field in every internal point of the scatterer, and which is accurate when the scattered field is much smaller than the incident field (typical case of soft tissues). The use of powerful computers makes it possible nowadays to introduce highly complex algorithms $[9,10]$, and many experimental devices have been developed based on linear, circular and/or toroidal arrays, and on mechanical and/or electronic steering and scanning [11, 12, 13, 14, 15]. When $U C T$ is applied to hard tissues like bones, the problems involved become more complex, mainly because of the high contrast existing between bone and the surrounding soft tissues [16]. However, several authors have developed algorithms by simply linearizing the inverse problem [17, 18, 19, 20, 21, 22] using both straight ray or Born approximations. However, difficulties arise with respect to quantitative tomography, i.e., for mapping the velocity or the attenuation of the ultrasonic waves, or the acoustical impedance of the medium. Finding solutions in these cases involves either using non-linear schemes [23] and/or performing extensive studies on the limitation of the approximations [24, 25].

In this chapter, we describe two iterative UCT methods based on the use of Born approximation. In both cases, the general scheme consists in estimating, at step n, a small deviation from a previously estimated configuration of the medium at step $\mathrm{n}-1$. At each step, the field deviation, induced by the medium inhomogeneity (also called the perturbation), is indeed related to that previous one by a linear relationship. For weakly inhomogeneous media, starting from a homogeneous reference (also called constant background) having properties near the mean acoustical properties of the medium, the strategy to solve the inverse problem necessitates only one iteration, and a first-order approximation is generally sufficient. Since in that case the field deviation (the perturbation) from the reference field (field in absence of the perturbation) is linearly related to the medium deviation (the medium perturbation) by a simple spatial Fourier transform, the inversion results in a simple inverse spatial Fourier transform which can be easily implemented by a filtered back-projection algorithm [26] like ones commonly used in X-ray $C T$ scanners. For more contrasted media several iterations are necessary.

When the problem can be reduced to the study of a fluid-like cavity (like marrow) buried in an elastic hollow cylinder (like bone) surrounded by fluid (like muscle), we propose an iterative method, based on broken straight rays taking into account the wave refraction at the bone-soft tissue interface $[7,25]$. This approach, called Compound Quantitative Ultrasonic Tomography (CQUT), is purely experimental and consists in performing reflection and transmission measurements, using an iterative correction procedure, which compensates for refraction effect arising at the boundary between bone and the surrounding tissues. The reflected/scattered waves provide information about the bone's geometrical properties, and the transmission waves, within a refracted straight ray approach, provide the local wave velocities. The tomographic reconstruction procedure is based on Born iterative method, and successive inverse spatial Fourier transforms. The main 
limitation of the CQUT method is the heavy experimental-costs involved (multiple iterative experiments).

The other $U C T$ method presented in this chapter is based on an iterative algorithm using successive high-order Born approximations. The tomographic method in this case is known as Distorted Born Diffraction Tomography (DBDT) [27]. In comparison with the previous upgrade, $D B D T$ combines diffraction measurements with a purely numerical non-linear inversion algorithm. This second approach is then more general than CQUT. It is not restricted to the case of a fluid-like cavity buried in an elastic hollow cylinder. Its main drawback is a heavy computational cost.

The performances and the limitations of these two tomographic methods applied to quantitative bone imaging problems are presented, and the results obtained using these methods are compared with experimental data.

\section{Ultrasonic Computed Tomography}

The aim of $U C T$ is to reconstruct the geometry and the spatial distribution of acoustical parameters of an object from scattered ultrasonic measurements.

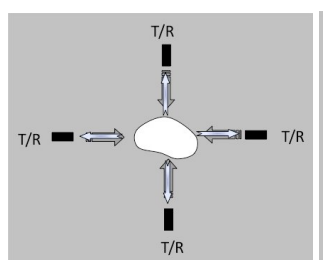

(a)

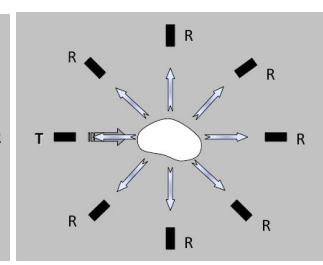

(b)

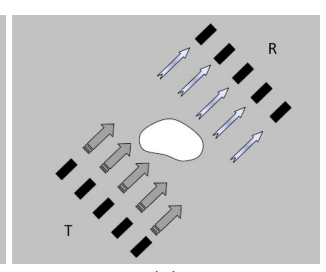

(c)

Fig.18.1. Operating modes in Ultrasonic Computed Tomography. (a) A single transmitter/receiver in the reflection mode, (b) " $n$ " transmitters/receivers in the diffraction mode, and (c) two paired transducers, one transmitter and one receiver in the transmission mode, are translated (the number of displacements corresponding to the points on the projections) and rotated around the tested body.

UCT measurements are carried out using variably densely spaced sets of transmitter and receiver positions as illustrated in Fig.18.1. The reconstruction of the object (geometry and acoustical properties) requires first a accurate model to solve the forward scattering problem, i.e. predicting the pressure field when the scattering medium and the incident field are assumed to be known, and second, solves the inverse scattering problem, i.e. determines the parameters of the medium from measurements of the incident and scattered fields on some surface. Inverse scattering problems are non-linear and ill posed. No single solution exists and it is necessary to find a way of eliminating the solutions that do not correspond to reality.

Basic UCT principles have been clearly established in the case of weakly varying media such as low-contrasted tissues. 
The forward scattering problem can be then solved with the Lippmann-Schwinger integral equation [23, 28], using the Green function [29] of the unperturbed problem (the homogeneous reference medium or constant background). Various approximations like straight ray approximation, for propagation measurements, or the first-order Born approximation, for scattering measurements, can be used in order to linearize the integral representation. This leads to a linear relation between the object function, which is related to the characteristics (dimensions, shape or acoustical parameters) of the reconstructed object, and the scattered field. Then, one possible way to solve this inverse problem consists in performing a far field asymptotic development [23], and 2-D or 3-D Fourier transforms, which makes it possible in principle to reconstruct the object function in almost real time based on a sufficiently large set of scattering data ("classical" tomographic algorithm) [26].

However, if the contrast between the media increases, the first-order Born approximation is no longer valid and other strategies will be considered. The first strategy adopted in this case consists in iteratively correcting the experimental data acquisition procedure, depending on the reflection and refraction behavior of the waves propagating through the soft/hard tissue interface. This strategy, which is known as Compound Quantitative Ultrasonic Tomography (CQUT), makes it possible to use the first-order Born approximation, correcting at each iteration, i.e. in each experiment, the refraction of the propagating wave due to the impedance contrast between the surrounding medium and the bone. The disadvantage of this procedure is that as many experiments as iterative steps have to be carried out.

A second strategy involves the algebraic inversion of the scattered field, based on the distorted Born iterative method, using iterative numerical steps and performing only one experiment.

\subsection{Modeling and linearization}

Let A be the operator that describes acoustic propagation or scattering phenomena in the heterogeneous medium that is to be imaged (including boundary and/or Sommerfeld radiation condition at infinity [23]). Let $\mathrm{S}$ be the acoustic sources, which are assumed to be known. All operators are time and space dependants. The variable $\varphi$, which denotes the resulting acoustic field, satisfies the equation:

$$
\mathrm{A} \varphi=\mathrm{S}
$$

Let us assume the medium to be composed of a known part, related to the reference medium, resulting in an operator $\mathrm{A}_{0}$, and an unknown part, related to the perturbation of the reference medium and identified by the object function, resulting in an operator $\mathrm{A}^{\prime}$ such that:

$$
\mathrm{A}=\mathrm{A}_{0}+\mathrm{A}^{\prime}
$$


Assuming that $\varphi_{0}$, the solution of the non-perturbed problem, is known:

$\mathrm{A}_{0} \varphi_{0}=\mathrm{S}$

Let $\varphi^{\prime}$ be the difference between $\varphi$ and $\varphi_{0}\left(\varphi^{\prime}=\varphi-\varphi_{0}\right)$, that is the field perturbation induced by the perturbation of the reference medium. Therefore $\varphi^{\prime}$ is the solution of

$\mathrm{A}_{0} \varphi^{\prime}=-\mathrm{A}^{\prime}\left(\varphi_{0}+\varphi^{\prime}\right)$

If the Green function $\mathrm{G}_{0}$ of the unperturbed problem is given [8], we obtain

$\varphi^{\prime}=\mathrm{G}_{0} \mathrm{~A}^{\prime}\left(\varphi_{0}+\varphi^{\prime}\right)$

The latter equation is the well-known Lippmann-Schwinger equation [23, 28], and the so-called inverse problem is therefore the solution of this non-linear equation. A solution can be found by using a perturbation scheme, based on successive linear approximations. The "Born series" is one of these schemes introducing different development orders [8, 29]. Within the first-order Born approximation, the field perturbation $\varphi^{\prime}$ is neglected in every internal point of the scatterer. The correspondent solution $\varphi_{1}^{\prime}$ can be written:

$\varphi_{1}^{\prime}=\mathrm{G}_{0} \mathrm{~A}^{\prime} \varphi_{0}$

In the frequency range ( $>3 \mathrm{MHz}$ ) of classical $U C T$ of weakly heterogeneous soft tissues, the reference medium is considered to be constant in first approximation, leading to an Inverse Born Approximation (IBA) method with a "constant background". The final objective is to obtain suitable images from scattered measurements $\left(\varphi^{\prime}\right)^{m}$, where the subscript $\mathrm{m}$ stands for “measurements". Rotating the transducers around the object and transmitting broadband pulses at each position can be handled using the same approach as in $X$-ray tomography [30]: it provides a slice-by-slice spectral coverage of the object spectrum (2-D-spatial Fourier transform, $\mathrm{F}_{2 \mathrm{D}}$ ):

$\mathrm{A}^{\prime}=\left(\mathrm{F}_{2 \mathrm{D}}^{-1}\right)\left(\varphi^{\prime}\right)^{m}$

where $F_{2 D}^{-1}$ designates the inverse 2-D-spatial Fourier transform. A reconstruction can therefore be performed using a classical algorithm of the summation of filtered back-projections [30]. 


\subsection{Limitations in the case of bone imaging}

Since the acoustic impedance of bone is highly contrasted with that of the surrounding medium, the ultrasound propagation is perturbed by wave refraction, attenuation and scattering. This results in the propagation of more complex waves, such as those occurring in elastic volumes (compressional and shear waves). The weak scattering hypothesis is therefore not realistic.

However, by adopting some assumptions, the field of application of UCT can be extended to bone imaging. If the object to be imaged can be modeled by a set of concentric isotropic homogeneous noncircular "fluid-like" media representing the homogenized surrounding tissues, bone and marrow, only compressional waves are taken into account. In the diagnostic frequency range of bone $Q U S(<3 \mathrm{MHz})$, the wavelength of compressional wave (propagating at velocities ranging between 2000 and $4000 \mathrm{~m} . \mathrm{s}^{-1}$ ) in cortical bone is typically greater than $1 \mathrm{~mm}$, which remains much larger that the typical size of bone microstructures. Therefore bone itself in the cortical shell can be assimilated to a weakly heterogeneous medium, and the ultrasonic wave propagation will be minimally disturbed. So, the Born approximation is satisfied in this area. The $I B A$ with a "variable background" can be used here; the background here being the set consisting of the homogeneous solid cylinder and the homogeneous fluid surrounding medium.

On the other hand, the wavelength in water ranges between 0.5 and $6 \mathrm{~mm}$, which remains smaller than the mean diameter $(\approx 10 \pm 2 \mathrm{~mm})$ of the bone. The product $k a$, where " $k$ " is the wave number and " $a$ " is the mean radius of the bone, ranges between 2 and 47, and the configuration is therefore non-resonant. The background can be defined in terms of the following two parts: a solid part (bone) without any hollow and the surrounding water (or soft tissue), and the perturbed part, i.e. the object to be reconstructed, namely the cavity (marrow-filled medullary canal). The algorithm of summation of the filtered back-projections can then be used with some signal processing refinements. Despite the artifacts and biases affecting the assessment of the shell thickness, the main result obtained will be a qualitative tomogram of the cavity, where the gray or color level sets are not referenced to a physical parameter.

\subsection{Compound Quantitative Ultrasonic Tomography (CQUT)}

The main difficulties, however, arise when attempting to provide quantitative tomographic images of acoustical wave parameters. Finding solutions in this case involves either using non-linear schemes or performing extensive studies on the limitation of the Born series. Following the same approach than in the section 2.1, we can write:

$\varphi_{1}^{\prime}=\mathrm{G}_{\mathrm{b}} \mathrm{A}^{\prime} \varphi_{\mathrm{b}}$ 
where $G_{b}$ is the appropriate Green function of the "variable background". $\varphi_{b}$ and $A_{b}$ are respectively the corresponding field and the corresponding operator such that:

$A_{b} \varphi_{b}=S$ and $G_{b} A_{b}=-I$

where I is the identity operator. This strategy can be applied iteratively:

$\varphi_{n}^{\prime}=\mathrm{G}_{\mathrm{b}}^{\mathrm{n}-1} \mathrm{~A}_{\mathrm{n}}^{\prime} \varphi_{b}^{n-1}$

where $G_{b}^{n}$ is the inhomogeneous Green function of the "variable background" distorted/adapted for every iteration step $\mathrm{n}$. This non-linear inversion scheme is called the distorted Born iterative (DBI) method. The reconstruction algorithm is therefore the same as the previous classical one, and the solutions are iteratively determined using Eq. 18.7. UCT based on the DBI method yields quantitative images.

Experimentally, the approach was designed first, to cancel out the refraction effects by using a specific set-up in order to impose straight ray propagation inside the shell, and second, to use the Born iterative method. Based on a priori knowledge of the geometrical properties and the acoustical properties, after calculating the incident and refracted angles using the Snell-Descartes laws, a compensation procedure has to be performed to determine the most suitable positions and orientations of the paired transducers (Fig.18.2).

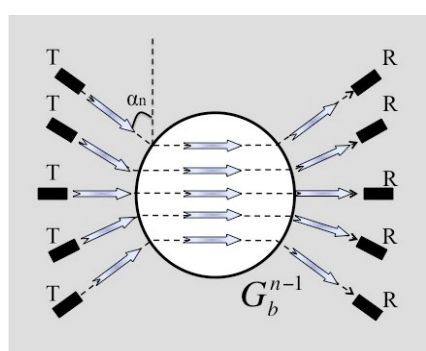

Fig.18.2. Compensatory operating procedure in $C Q U T-\alpha_{n}$ is the incident angle determined at the step $n$ using the Snell-Descartes laws; $G_{b}^{n-1}$ is the Green function adapted to a variable background [homogeneous cylinder plus homogeneous surrounding medium] at each iteration.

Reflection measurements and transmission measurements give, respectively and successively, the boundaries of the shell and the quantitative values of the velocity of the wave along the whole path. A quantitative image obtained after experiment $n$ is used as the a priori information for the following procedure $n+1$. The initial guess is the image obtained without any angular corrections, and the stop criterion 
of this iterative process is when the difference between the mean velocities calculated at two different steps is less than $5 \mathrm{~m} \cdot \mathrm{s}^{-1}$.

Our initial attempts along these lines have been improved using signal and image processing methods [31]. This iterative experimental method, known as Compound Quantitative Ultrasonic Tomography (CQUT), has been described in detail in $[32,33]$. Despite limitations due to heavy data processing requirements and complex acoustical signals resulting from multiple physical effects involved (various pathways into the shell, roughnesses of the water/bone interfaces etc... [34]), CQUT gives images that are quantitatively related to the compressional wave velocities in a cross-section of a cortical shell, and the error remains within reasonable limits (about 7\%).

\subsection{Distorted Born Diffraction Tomography (DBDT)}

A second non-linear inversion method, which is also based on the $D B I$ method, was investigated. With this approach, the medium is modeled without any a priori knowledge by performing a simple geometrical discretization of the object. The algorithm involves successive linearizations of the Lippmann-Schwinger representation. The initial guess in the iterative process is provided by the firstorder Born approximation. If the solution is known with the order $(\mathrm{n}-1)$, the $\mathrm{n}$ order solution $A_{n}^{\prime}$ will satisfy [35]:

$$
\left[\left(\varphi^{\prime}\right)^{m}-\varphi_{n-1}^{\prime}\right]=G_{b}^{n-1}\left[A_{n}^{\prime}-A_{n-1}^{\prime}\right] \varphi_{b}^{n-1}
$$

At each iteration, the algorithm numerically solves a forward diffraction problem in order to calculate the appropriate inhomogeneous Green function $G_{b}^{n-1}$ and the internal field $\varphi_{b}^{n-1}$. Contrary to what occurs with the CQUT, the $D B D T$ requires only a single series of experimental data. However, this technique is computational time consuming because it involves inversion of huge, full and complex matrix. The matrix inversion procedure is the key point with this method. Generally non-square ill-conditioned matrixes have to be inverted. A mean-square solution can be calculated using a conjugated-gradient method associated with a regularization procedure [36]. To make use of the broadband frequency content of the impulse signal used, the idea is to begin with the low frequencies, which carry overall information, and to gradually inject the high frequencies to simultaneously improve both the qualitative aspects (the resolution) and the quantitative aspects (the characterization). 


\section{The $U C T$-scanner}

These methods were tested on data obtained with a mechanical scanner, on bonemimicking phantoms and real human bones. Whenever possible, UCT images of bones were compared with $X$-ray tomography images obtained at the same crosssection levels. Details of the imaging conditions are systematically presented below.

The general architecture of this mechanical system is that of a first generation ultrasonic scanner (Fig.18.3): the main symmetrical arm holds two transversal arms with which two transducers can be translated in parallel. By rotating either the main arm or the object holder, angular scanning can be performed. Six stepping motors sequentially driven by a programmable translator-indexer device fitted with a power multiplexer control all the movements. The object to be imaged is placed in the presumed geometrical center of the bench so that the distance between the transducers and the center is limited to $150 \mathrm{~mm}$. The surrounding fluid-like medium is water at a temperature of $18.6^{\circ}\left(\rho_{\mathrm{o}}=1000 \mathrm{~kg} \cdot \mathrm{m}^{-}\right.$ $\left.{ }^{3}, \mathrm{c}_{\mathrm{o}}=1480 \mathrm{~m} \cdot \mathrm{s}^{-1}\right)$. The transducers used for data acquisition are broadband piezocomposite transducers with nominal frequencies of $250 \mathrm{kHz}$ to $1 \mathrm{MHz}$. The transducers are driven using a pulse/receiver, and are positioned automatically as required around the object, and the data stored are then used to determine the time-of-flight between the source, the object and the receiver. The reconstruction algorithm and some signal processing algorithms were implemented on a personal computer.

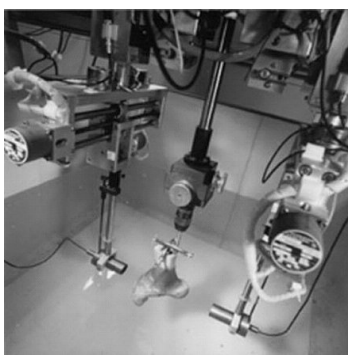

(a)

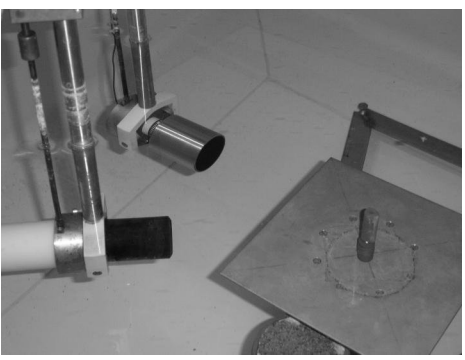

(b)

Fig.18.3. The $U C T$-scanner, (a) transmission configuration, (b) reflected (only one transducer is used) and diffracted (two transducers are used) configurations 


\section{Results}

\subsection{D and 3D qualitative tomography}

Contrast tomographic images of bone were obtained using the linearized (firstorder Born approximation) UCT algorithm based on reflected and diffracted measurements (no transmission measurements were used at this stage). Except for the processing of the input signals, no compensatory procedures or changes in the wave paths were made.

\subsubsection{Lumbar vertebrae}

The first example focuses on the analysis of a L2 lumbar human vertebra without any articular or transversal apophysis, having a visible external spinal body diameter of approximately $30 \mathrm{~mm}$ (Fig.18.4a). A 4-mm circular metallic rod was placed inside the specimen, perpendicular to its upper surface.

The nominal frequency of the transducer (Fig.18.4b) was $500 \mathrm{kHz}(\lambda=3 \mathrm{~mm}$ in water) and the reflected sinogram consisted of 180 projections (through $360^{\circ}$ ), each including 1024 samples. The sampling frequency was $20 \mathrm{MHz}$. The size of the image was $255 \times 255$ pixels. The resolution was improved using Papoulis deconvolution of the signal measured by the transfer function of the apparatus, with a frequency threshold of $-15 \mathrm{~dB}$ in the [330-760] $\mathrm{kHz}$ frequency range [37]. Under these signal-processing conditions, the resolution of the image was about $0.375 \mathrm{~mm}(\lambda / 8$ in water $)$.

The $X$-ray tomography device (Fig.18.4c) was a Philips MG 450 radiation source, with a high intensity tube receiving a $80 \mathrm{keV}$ beam with an intensity of $10 \mathrm{~mA}$. The focal size was $4.5 \mathrm{~mm}$. The distance from source to object was $3 \mathrm{~m}$. A Thalès Flashscan 35 was used as the scintillator-imaging device. The resolution was 127 $\mu \mathrm{m}$, and the image size was 2304 x 3200 pixels.

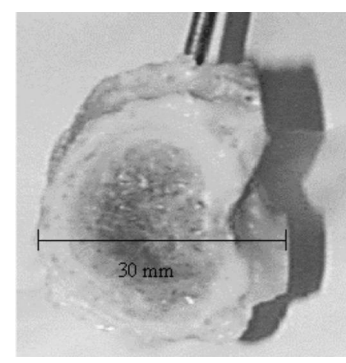

(a)

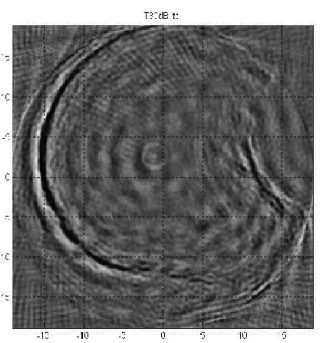

(b)

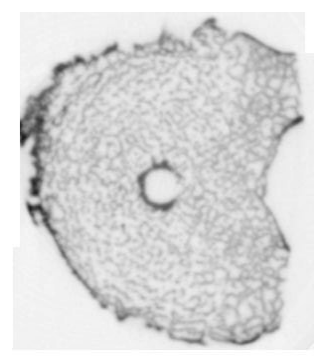

(c)

Fig.18.4. L2 -Lumbar vertebra. (a) Sample picture, (b) 2-D-UCT obtained from the reflected sinogram, 180 projections with 1024 samples, Nominal frequency $\mathrm{Fc}=500 \mathrm{kHz}$, 
resolution $375 \mu \mathrm{m}$, image size 255 x 255 pixels (c) corresponding $X$-ray tomography, resolution $127 \mu \mathrm{m}$, image size 2304 x 3200 pixels.

The dimensions and the shape of the bone sample could be readily distinguished on the UCT image. The dimensions and the location of the rod were also visible and well reconstructed. This means that on the one hand, the ultrasonic wave propagated into the center of the body despite the attenuation of the wave due to the porosity and/or the anisotropy, and that on the other hand, it was then possible to discriminate and to size a metallic implant placed inside the bone specimen. But it was impossible to discriminate between the trabecular zone and the cortical zone. In addition, $U C T$ does not give the same quality of image resolution as $X$-ray tomography. This is a serious limitation, as it makes it difficult to determine some characteristic parameters precisely from $U C T$ images, such as the bone volume fraction.

\subsubsection{Diaphysis of an adult thighbone}

In the second example, a 3-D UCT of adult human female thighbone was obtained from diffracted sinograms. The first sample (Fig.18.5) was obtained from a postmenopausal 78-year old woman with osteoporosis, and the second (Fig.18.6) from a 81-year-old woman without any bones pathology (healthy bones).

The nominal frequency of the transducers was $1 \mathrm{MHz}$. The resolution and the size of the image were $0.75 \times 0.75 \mathrm{~mm}$ and 512 x 512 pixels, respectively. The diffracted sinograms included 2048 projections, each including 8256 samples, involving 32 angular transmitter positions combined with 64 angular receiver positions covering an angle of $360^{\circ}$. The sampling frequency used was $40 \mathrm{MHz}$. A signal-processing tool $[20,38]$ was used to determine cortical thickness. This tool was based on a segmentation of the final image and a size correction of the inner boundaries, using a priori information on physical parameters (in this case, mean compressional wave velocity in bone $=3500 \pm 100 \mathrm{~m} \cdot \mathrm{s}^{-1}$, bone mass density $=1700 \mathrm{~kg} \cdot \mathrm{m}^{-3}$ ).

Fig.18.5a and Fig.18.6 show the 3-D UCT images of the pathological and healthy thighbones. These reconstructions were obtained by superimposing sequential $2-D$ UCT images (80 cross-sections). The step between two cross-sections was 0.25 $\mathrm{mm}$. The interpolation scheme used for a given 3-D image was the shape-based method (Matlab®, MathWorks ${ }^{\mathrm{TM}}$ ). In Fig.18.5b and Fig.18.5c, three 2-D UCT spaced 6 and $4 \mathrm{~mm}$ apart $(\mathrm{H} 1=8 \mathrm{~mm}, \mathrm{H} 2=14 \mathrm{~mm}$ and $\mathrm{H} 3=18 \mathrm{~mm})$ are compared with the corresponding $X$-ray tomographic images of the unhealthy bone.

The $X$-ray device was a clinical General Electric ${ }^{\circledR}$ device $(C E 12000)$. The thickness of the cross-section was $1 \mathrm{~mm}$. The resolution was $0.25 \times 0.25 \mathrm{~mm}$ and the image size was $512 \times 512$ pixels. 


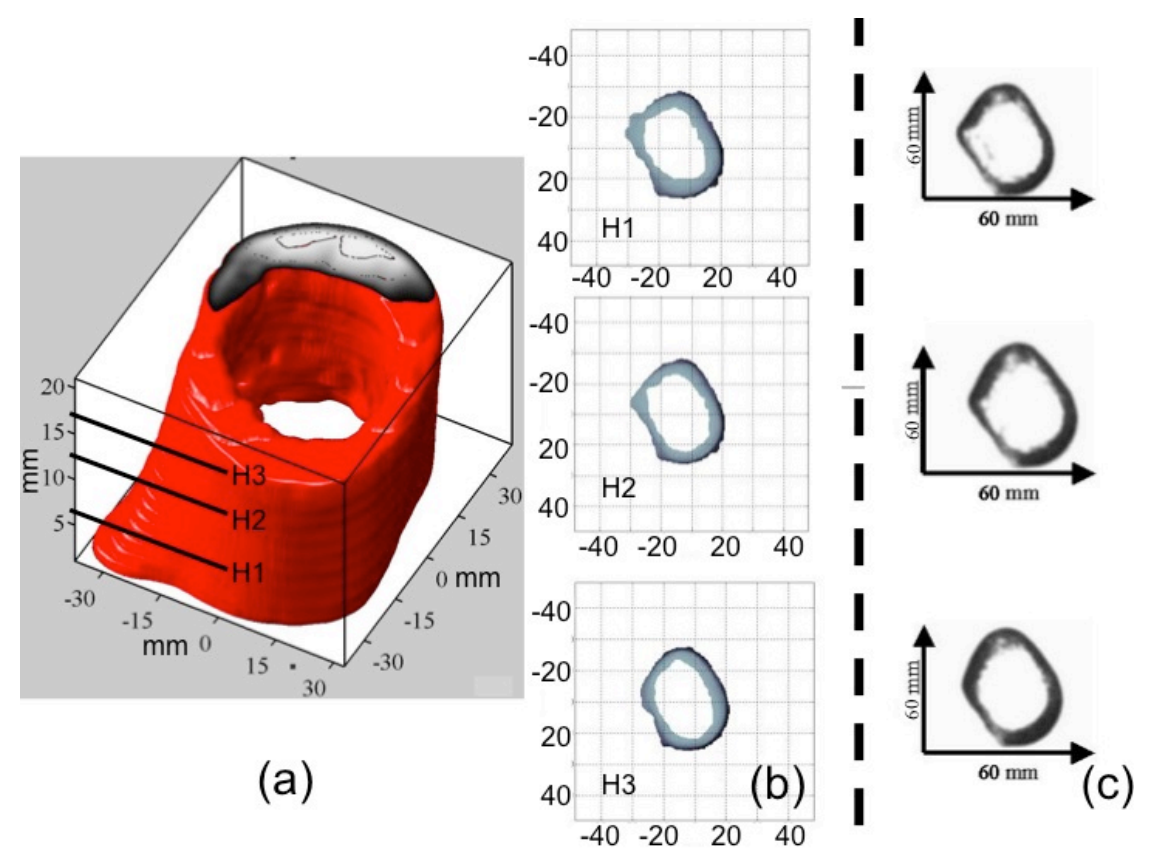

Fig.18.5. Diaphysis of an adult thighbone with osteoporosis. (a) Qualitative image obtained with 3-D UCT, (b) cross-sections, $\mathrm{H} 1=8 \mathrm{~mm}, \mathrm{H} 2=14 \mathrm{~mm}$ and $\mathrm{H} 3=18 \mathrm{~mm}$, (c) corresponding $X$-ray tomographies.

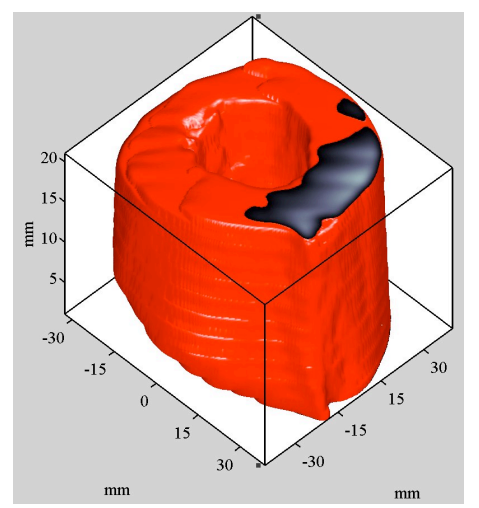

Fig.18.6. 3-D-UCT of a healthy thighbone

\subsubsection{Childhood fibula}

In the third example (Fig.18.7), the same experimental configuration was used as previously (see subsection 4.1.2). The sample was a fresh fibula from a 12-year old child containing no marrow in the inner cavity. The mean dimensions of the 
bone sample were $17 \pm 2 \mathrm{~mm}$ on the outside and $6 \pm 2 \mathrm{~mm}$ in the inner cavity. Twenty sequential cross-sections were performed in this case with a 1-mm step.
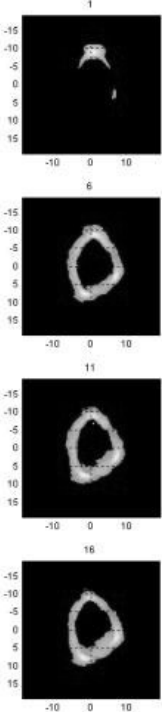
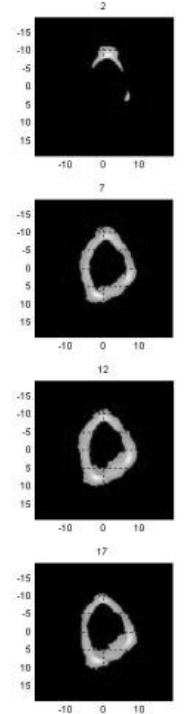
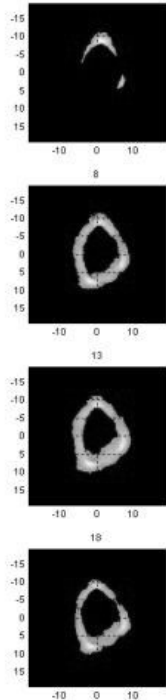
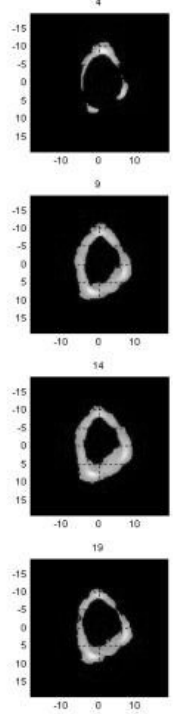
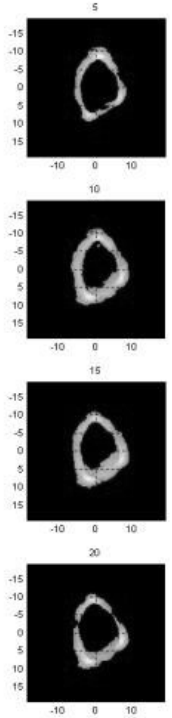

Fig.18.7 3-D UCT image of a child's fibula

These results obtained with human specimens show that it is possible to characterize the size and shape of bones using Ultrasonic Computed Tomography. Focusing on well-contrasted images obtained using $U C T$, and comparison with $X$ ray, show that this method provide efficient means of assessing the cortical thickness, which is known to be an important indicator of bone strength.

\subsection{Quantitative assessment}

The aim of the second experiment was to obtain a quantitative assessment of the ultrasonic wave velocity in bone using $U C T$ methods.

\subsubsection{Compound Quantitative Ultrasonic Tomography (CQUT)}

CQUT was tested first on circular Plexiglas cylinders and results were excellent because of the weak errors and the fast convergence of the algorithm (only 2 iterations) [33]. Because long bones look like more non-cylindrical elastic hollow tubes, the number of iterations (about 5 here) is higher and the convergence is slower than with academic objects [32]. 
The bone sample tested here was a human femoral diaphysis with an external diameter of about $32 \pm 5 \mathrm{~mm}$ and an internal diameter of $16 \pm 2 \mathrm{~mm}$. The initial velocity of the compressional wave propagating into the bone was set at $3400 \mathrm{~m} . \mathrm{s}^{-}$ 1 .

In the $U C T$, the backscattered sinogram consisted of 90 signals covering an angle of $360^{\circ}$. The transmitted sinogram consisted of 90 projections with 128 transverse displacements in steps of $330 \mu \mathrm{m}$. The time-of-flight was calculated with several algorithms (zero-crossing, cross-correlation, etc.). The $X$-ray device used was that described above in section 4.1.1.

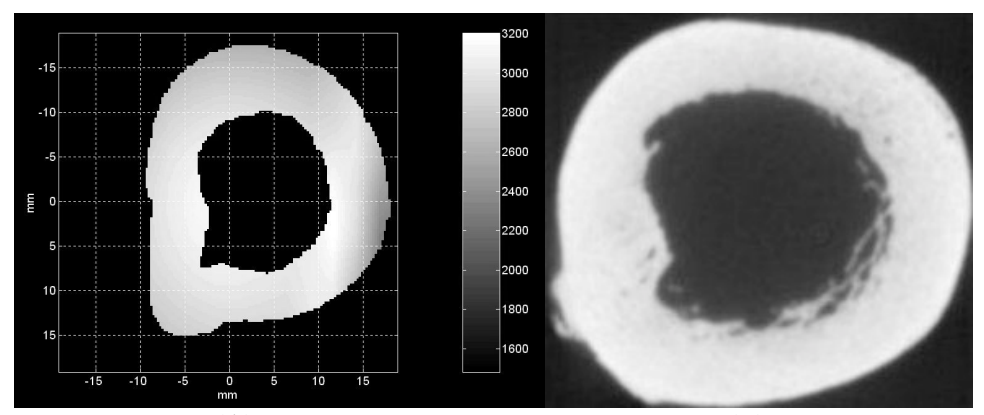

(A)

(B)

Fig.18.8. Human thighbone (A) CQUT (90 projections, 128 translation samples; resolution $0.75 \times 0.75 \mathrm{~mm}$, size $256 \times 256$ pixels), (B) corresponding $X$-ray tomography ( $80 \mathrm{keV}, 10 \mathrm{~mA}, 4.5 \mathrm{~mm}$, resolution $127 \mu \mathrm{m}$, size 2304 x 3200 pixels)

From the qualitative (contrast) point of view, the $U C T$ image was similar to the $X$ ray image (Fig.18.8). $X$-ray tomography, which estimates the mass density, is the main method currently used to determine the structural characteristics of bone, and the present reconstruction was more detailed than that obtained with UCT. However, the ultrasonic image represents a quantitative map of the velocity of the compressional wave into the cortical shell. The speed of sound of the fluid contained in the inner cavity was accurately reconstructed with a mean wave velocity close to $1500 \mathrm{~m} \cdot \mathrm{s}^{-1}$, and the diameter of the inner cavity was found to be $15-17 \mathrm{~mm}$. The external diameter was found to be in the $30-34 \mathrm{~mm}$ range, which is close to the actual values. Mean wave velocity in the shell was $3150 \pm 50$ $\mathrm{m} . \mathrm{s}^{-1}$. This value seems to be lower than the mean wave velocity in cortical bones usually reported in literature (see chapter 13 for more details). However, experimental studies were performed in parallel on small cubic samples taken from the same femur, and similar values with only a slight dispersion $\left(<0.5 \mathrm{~m} . \mathrm{s}^{-1}\right)$ were obtained.

\subsubsection{Distorted Born Diffraction Tomography (DBDT)}

The performances obtained with $D B D T$ were then assessed with a set of experimental data. The sample used here was a geometrically-mimicking phantom of a child's bone. The phantom was a non-circular homogeneous isotropic tube 
made of artificial resin (Neukadur ProtoCast $113^{\mathrm{TM}}$ ) with maximum internal and external diameters of 6 and $12 \mathrm{~mm}$, respectively. The volumetric mass density of the resin was $\rho_{1} \approx 1150 \mathrm{~kg} \cdot \mathrm{m}^{-3}$, and the mean velocity of the compressional wave was $\mathrm{c}_{1} \approx 2400 \pm 50 \mathrm{~m} \cdot \mathrm{s}^{-1}$. The transmitter and receiver were placed $17.5 \mathrm{~cm}$ to the right of the center of the bench. The diffraction sinograms were assessed with two transducers, which were placed in 72 by 72 positions around the object with a $5^{\circ}$ step. The transducers had a nominal frequency of $250 \mathrm{kHz}$ and their $-6 \mathrm{~dB}$ frequency bandwidth was $135-375 \mathrm{kHz}$. The initial frequency was chosen so that the fist-order Born solution, that provides the initial guess of the iterative process, does not include too many artifacts. Previous studies $[35,39]$ have shown that if the phase shift resulting from the presence of the scatterer is greater than $\pi$, the reconstruction will present important artifacts. We therefore chose an initial frequency such as:

$$
f<\frac{c_{0} c_{l}}{2 d\left|c_{l}-c_{0}\right|} \approx 150 \mathrm{kHz} \quad(18.12)
$$

where $\mathrm{d}$ is the largest dimension of the scatterer. Because of the small frequency range available, the image resolution rather limited. The scattered field was obtained by subtracting the incident field (measured without the scatterer) from the total field. The frequency data were obtained by computing the Fast Fourier Transform of the temporal signals and no other corrections or signal processing steps were carried out.

Fig.18.9a shows the first-order diffraction tomography of the scatterer. Since the wavelength of the wave in water was similar to the size of the object, the image resolution is poor and the assessment of the shell thickness is not possible.

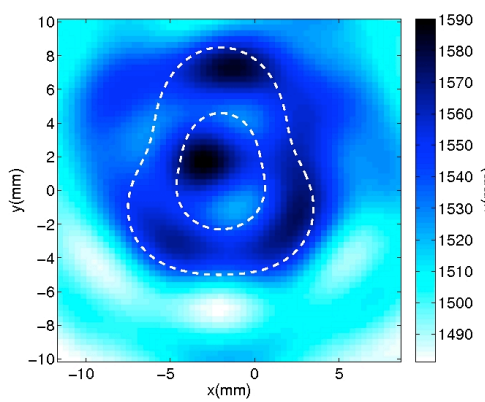

(a)

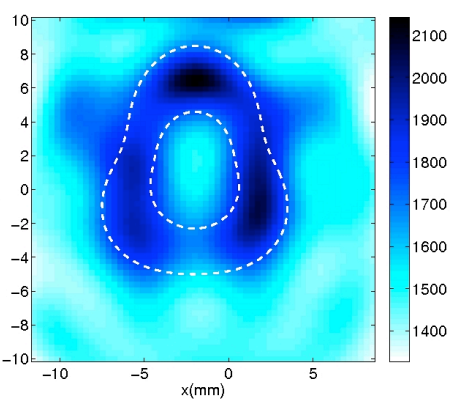

(b) 


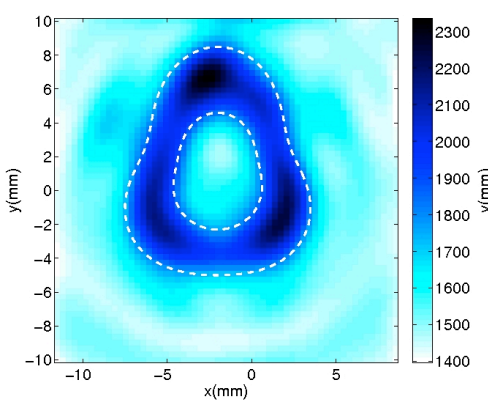

(c)

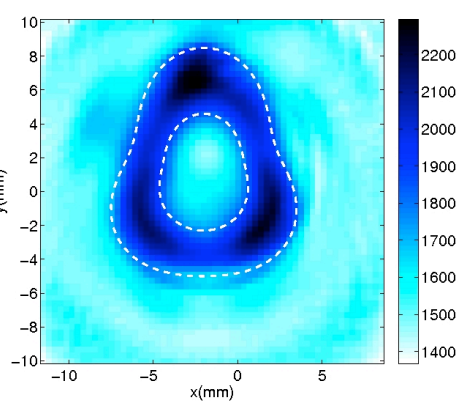

(d)

Fig.18.9. Quantitative $U C T$ of a geometrically-mimicking phantom of a child's bone. (a) Initial solution of the $D B D T$ at $150 \mathrm{kHz}$ (first-order Born approximation), (b) iterations at $150 \mathrm{kHz}$, (c) iterations at $350 \mathrm{kHz}$, (d) iterations at $1 \mathrm{MHz}$.

Fig.18.9b, Fig.18.9c and Fig.18.9d show three sequential iterations of the distorted Born diffraction tomography. It can be seen that with $D B D T$, the resolution and the quality of the contrast gradually improved. The final result of the iteration process was satisfactory. The geometry was fairly accurate (position and dimensions reconstructed with mean relative error of the order of 5\%), whereas the velocity was estimated with a rather large relative error of about $10 \%$.

\section{Conclusion}

This chapter deals with 2-D and 3-D imaging of human long bones using Ultrasonic Computed Tomography (UCT). The scope of UCT methods was thus extended here from low impedance and velocity contrast media such as soft tissues (the classical domain) to higher contrast domains such as cortical bone structures, using non-linear and correction schemes with the following features: the wave field and the associated Green function of the reference background medium were determined iteratively at the various steps. Due to the mismatch between the acoustical impedance of bone and that of the surrounding soft tissues, the higher the frequency, the lower the proportion of the energy transmitted through bone and the lower the resolution of the resulting ultrasonic image. The ultrasonic propagation is greatly perturbed by the contrast between the media, which generates large artifacts. Two strategies were used to solve this problem.

The first strategy, named Compound Quantitative Ultrasonic Tomography (CQUT), was based on the Born iterative method with a corrected experimental data acquisition procedure. Bone was assumed to be equivalent to an internally weakly contrasted object immersed in a homogeneous reference medium (water). The results obtained with CQUT were satisfactory, and both the reconstructed geometries and wave velocities were close to the actual values. The main limitations of the CQUT are the number of measurements required, which involve multiple iterative experiments and heavy data processing. 
The second method tested here was also a non-linear inversion method with higher-order levels of approximation, but in this case, the iterations were performed numerically, based on a single experimental measurement. The socalled Distorted Born Diffraction Tomography (DBDT) strategy gave reasonably accurate results without requiring any a priori information about the object.

The methods presented in this chapter were tested so far using bone mimicking phantoms as well as real bones, including vertebrae, femurs and fibulas, with a non-canonical homogeneous shape. In comparison with classical $X$-ray tomography, UCT methods were found to be promising, and the geometrical and physical parameters of the object were accurately reconstructed with these methods.

It is now proposed to investigate various ways of improving these methods. Work is in progress, for instance, on the matrix inversion procedure involved in $D B D T$ method and particularly on the regularization process, which is an extremely important aspect of the inversion scheme, especially with high-contrast targets. Signal processing and image processing studies on how to handle the heavy experimental data are also in progress (wavelet analysis, blind deconvolution, segmentation etc...).

In conclusion, in situ measurements of the acoustic properties of long bones in various parts of the skeleton must be sufficiently accurate for the results to be of use in the diagnosis and/or treatment of bone diseases. Once the requisite degree of accuracy has been achieved, it will be possible to start developing prototypes for in vivo applications.

Acknowledgments The authors are grateful for medical assistance from Dr P. Petit and Dr J.-L. Jouve at Public Assistance Hospitals in Marseille and the "Timone" Children's Hospital. The $X$ ray tomographies were performed by V. Kaftandjian at the Laboratory for Nondestructive Testing using Ionizing Radiation, INSA, Lyon.

1 Smith S.W., Von Ramm O.T, Kisslo J.A, Thurstone F.L (1978) Real time ultrasound tomography of the adult brain. Journ. of the American Heart Association, Stroke, 9:117-122

2 Migeon B., Marche P. (1994) 3D surfacing representation of limb long bones using ultrasound tomography. ITBM 15(6):693-706

3 Greenleaf J.F. (1970) Introduction to computer ultrasound tomography. Computed Aided Tomography and Ultrasonics in Medicine, North-Holland

4 Norton S.J., Linzer M. (1979) Ultrasonic reflectivity imaging in three dimensions: reconstruction with spherical transducer arrays. Ultrasonic Imaging 1:210-231

5 Lefebvre J.P. (1986) Acoustic Impedance Tomography: two reconstructions procedure. Acoustical Imaging 14:719-723 

diffraction computed tomography for breast imaging: studies in phantoms and patients. Acoustical Imaging 21:379-390

7 Anderson A.H., Kak A.C. (1984) The application of ray tracing towards a correction for refracting effects in computed tomography with diffracting sources. School of Electrical Engineering, Perdue University (Ed), West Lafayette Indiana 47907

Born M., Wolf E, (1964) Principles of optics. In: Pegamon Press (Ed), Oxford.

9 Mensah S., Lefebvre J.P. (1997) Enhanced compressibility tomography. IEEE Trans. Ultrason. Ferroelec. Freq. Contr. 44(66):1245-1252

10 Franceschini E., Mensah S., Le Marrec L., Lasaygues P. (2007) An optimization method for quantitative impedance tomography. IEEE Trans. Ultrason. Ferroelec. Freq. Contr. - Special Issue on High Resolution Ultrasonic Imaging in Industrial, Material and Biomaterial Applications 54(8):1578-1588

11 André M.P., Janée H.S., Martin P.J., Otto G.P., Spivey B.A., Palmer D.A. (1997) High-speed data acquisition in diffraction tomography system employing large-scale toroidal arrays. International Journal of Imaging System and Technology 8(1):137-147

Johnson S.A., Borup D.T., Wiskin J.W., Natterer F., Wubbling F., Zhang Y. , Olsen C. (1999) Apparatus and method for imaging with wavefields using inverse scattering technique. US Patent 6, 005, 916

13 Lasaygues P., Tanne D., Mensah S., Lefebvre J.P. (2002) Circular antenna for breast ultrasonic diffraction tomography. Ultrasonic imaging 24:135-146

14 Duric N., Littrup P., Babkin A., Chambers D., Azevedo S., Arkady K., Pevzner R., Tokarev M., Holsapple E. (2005) Development of ultrasound tomography for breast imaging: technical assessment. Medical Physics 32(5):1375-1386

Waag R.C., Fedewa R.J. (2006) A ring transducer system for medical ultrasound research. IEEE Trans. Ultrason. Ferroelec. Freq. Contr. 53(10):1707-1718

16 Lefebvre J.P., Lasaygues P., Mensah S. (2000) Born ultrasonic tomography: some limits and improvements. Acoustical Imaging 25:79-86

17 Carson P.L., Oughton T.V., Hendee W.R. (1976) Ultrasound transaxial tomography by reconstruction. In: Plenum Press, White D.N. and Barnes R.W. (ed) Ultrasound in Medicine II

Carson P.L., Oughton T.V., Hendee W.R., Ahuja A.S. (1977) Imaging soft tissue through bone with ultrasound transmission tomography by reconstruction. Medical Physics 4(4):302309

19 Merk H. (1988) Ultrasonic tomography in the diagnosis of diseases of the skeletal system in childhood. Radiol. Diagn. 29(2):269-278

20 Ylitalo G., Koivukangas J., Oksman J. (1990) Ultrasonic reflection mode computed tomography through a skullbone. IEEE Transactions on Biomedical Engineering 37(11): 1059-1066

21 Sehgal C.M., Lewallen D.G., Greenleaf J.F., Robb R.A., Nicholson J.A. (1988) Ultrasound transmission and reflection computerized tomography for imaging bones and adjoining soft tissues. IEEE Ultrasonics Symposium 2: 849-852 
23 Chew W.C. (1995) Waves and fields in Inhomogeneous Media. In: IEEE Press (ed), NewYork.

24 Slaney M., Kak A.C., Larsen L. (1984) Limitations of imaging with first-order diffraction tomography. IEEE Trans. Microwave Theory Tech. 32:860-874

Teng X. (1993) Application of ultrasonic tomography in industrial materials. Ph.D. Thesis The John Hopkins University

Lefebvre J.P. (1988) A linearized inverse problem: acoustic impedance tomography of biological media. In: Bourelly $\mathrm{C}$. and al (ed) Electromagnetic and acoustic scattering: detection and inverse problem

Lu C., Lin J., Chew W.C., Otto G. (1996) Image reconstruction with acoustic measurement using distorted Born iteration method. Ultrasonic Imaging 18(2):140-156

Ishimaru A (1991) Electromagnetic wave propagation, radiation, and scattering. Englewood Cliffs, NJ: Prentice-Hall

Morse P M, Feshbach H (1953) Methods of mathematical physics. In: McGraw-Hill (Ed), New York.

30 Kak A.C., Slaney M. (2001) Principles of computerized tomographic imaging. In: Society for Industrial and Applied Mathematics Ed, Philadelphia, PA

31 Lasaygues P., Lefebvre J.P., Mensah S. (1998) Deconvolution and wavelet analysis on ultrasonic reflection tomography. In: Maldague X. (ed) Advances in Signal Processing for NDE of Materials, ASNT 3:27-32

Ouedraogo E., Lasaygues P., Lefebvre J.P., Gindre M., Talmant M., Laugier P. (2001) Multistep compensation technique for ultrasound tomography of bone. Acoustical Imaging 26:153160

33 Ouedraogo E., Lasaygues P., Lefebvre J.P., Gindre M., Talmant M., Laugier P. (2002) Contrast and velocity ultrasonic tomography of long bones. Ultrasonic Imaging 24:135-146

34 Lasaygues P. (2007) Compound quantitative ultrasonic tomography of long bones using wavelets analysis. Acoustical Imaging 28:223-229

Haddadin O. S. (1998) Ultrasound inverse scattering for tomographic imaging self-focusing array. Ph. D. Thesis University of Michigan

36 Lasaygues P., Guillermin R., Lefebvre J.-P. (2006) Distorted Born diffraction tomography applied to inverting ultrasonic field scattered by noncircular infinite elastic tube. Ultrasonic Imaging 28(4):211-229

37 Lasaygues P., Lefebvre JP. (2001) Cancellous and cortical bone imaging by reflected tomography. Ultrasonic Imaging 23:55-68.

38 Lasaygues P. (2006) Assessing the cortical thickness of long bone shafts in children, using two-dimensional ultrasonic diffraction tomography. Ultrasound in Med. \& Biol. 12(8):12151227 
Haddadin O.S., Ebbini E.S. (1998) Imaging strongly scattering media using a multiple frequency distorted Born iterative method. IEEE trans on Ultrasonics, Ferroelectrics and Frequency Control. 45:1485-1496 